\title{
Ultrasound-assisted Water-Confined Laser Micromachining (UWLM) of Metals: Experimental Study and Time-Resolved Observation
}

\author{
Ze Liu ${ }^{\mathrm{a}}$, Benxin $\mathrm{Wu}^{\mathrm{a} *}$, Avik Samanta ${ }^{\mathrm{b}}$, Ninggang Shen ${ }^{\mathrm{b}}$, Hongtao Ding ${ }^{\mathrm{b}}$, Rong Xu${ }^{\mathrm{a}}$, and Kejie Zhao ${ }^{\mathrm{a}}$

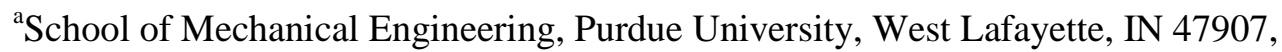 \\ ${ }^{\mathrm{b}}$ Department of Mechanical and Industrial Engineering, The University of Iowa, Iowa City, IA 52242
}

\begin{abstract}
This paper reports the experimental studies and time-resolved observations of the ultrasound-assisted water-confined laser micromachining (UWLM) process on metal workpieces using 532-nm nanosecond laser pulses. UWLM is a new machining process proposed by the corresponding author (Wu, 2014). During UWLM, a laser beam interacts with a workpiece's front surface location immersed in water to ablate the workpiece, and in-situ ultrasonic waves in water are also applied (for example, from above the front surface using an ultrasonic horn) to the laser ablation site (which energize the water and generate cavitations) to improve the machining process. This paper reports the experimental study of the UWLM process on multiple types of metal workpieces and time-resolved in-situ observations of the process. This kind of study on UWLM has been rarely reported in the literature. Under the studied conditions, it has been found that the ablation depths produced by UWLM at 200 laser pulses are about 2.3 to 3.8 times the depths by laser ablation in water without ultrasound. Through in-situ time-resolved shadowgraph imaging observations, it has been found that one important likely underlying mechanism for the enhanced material removal rate in the studied UWLM process is that the in-situ ultrasound in UWLM can reduce the material cloud resulted from the previous laser pulse ablation that lingers around laser ablation site, which can enhance the coupling of the subsequent laser pulse(s)' energy to the workpiece.
\end{abstract}

Keywords: Laser ablation, laser machining, laser micromachining, ultrasound

\section{Introduction}

Numerous fields have a need of micro-scale products, or products with critical microscale features, which may require micro-manufacturing technologies, such as a micromachining process. Ehmann (2007) gave a synopsis of the research and development activities in the micromanufacturing area in the United States. Masuzawa (2000) gave a review on the state of the art

\footnotetext{
* Corresponding author: Associate Professor, School of Mechanical Engineering, Purdue University, 585 Purdue Mall, West Lafayette, IN 47907, email: wu65@purdue.edu; Adjunct Associate Professor, Department of Mechanical, Materials and Aerospace Engineering, Illinois Institute of Technology, Chicago, IL 60616, email: bwu11@iit.edu.
} 
in the micromachining field. Laser micromachining is one of the existing micromachining technologies, where laser beam energy is delivered to a workpiece to remove materials and create or modify micro-scale features. Unlike mechanical micromachining, laser micromachining does not involve mechanical tool contact with workpieces, and hence does not have any related mechanical tool wear problem, or machining forces due to the mechanical tool-workpiece contact. The latter permits the laser spot to move very quickly on the workpiece (e.g., through a lase scanner). As a result, although the volume of materials removed per laser pulse is often small, laser micromachining still has a competitive productivity (as compared with mechanical micromachining, such as mechanical micro-milling) for many micromachining applications, where a large number of micro-features at numerous locations need to be produced, such that tool lifting, moving and dropping processes consume a sufficiently large fraction of the production time if a mechanical micro-milling approach is used. Laser micromachining trajectories can also be flexibly controlled and programmed. A laser beam can be focused down to a few micrometers (or even smaller) to machine very small features with high spatial resolutions. Dahotre and Harimkar (2008) gave a detailed introduction about laser machining.

Despite the aforementioned advantages, laser micromachining often suffers from defects, such as debris deposition, heat affected zone (HAZ), or other thermally induced detrimental effects. For example, Matsumura et al. (2005) studied the debris generation in silicon machining using femtosecond laser pulses, and revealed a related debris formation mechanism through small particle aggregation. Chien and Hou (2007) studied the recast layer generated during laser drilling of Inconel 718, and revealed some process parameters that greatly affect the thickness of the recast layer, which include the pressure of the assist gas during the process, laser beam peak power, and laser beam focal position 
One approach to potentially decrease one or more of the above defects is to immerse the workpiece surface (or at least the laser ablation site on the surface) in water during laser micromachining. Typically, water has a much higher thermal conductivity than a gas medium, and hence may produce a cooling effect to the workpiece. The water medium may also help decrease debris deposition (i.e., produce a cleaning effect). Investigations on laser machining in water have been reported in the literature. Kruusing (2004) gave a review of some research work in this area, which found that the application of water during laser machining can often reduce defects such as debris re-deposition and cracks. Morita et al. (1988) investigated laser drilling of silicon nitride ceramic in air and water environments, and they found that the former process led to cracks and recast layer in the workpiece, which were not observed in the latter process. Tangwarodomnukun et al. (2010) studied the micromachining of silicon by dry laser machining and underwater laser machining, and they found that the latter process can significantly reduce HAZ. Lv et al. (2016) have studied laser drilling of Inconel 718 alloy in water using 8-ns laser pulses at the wavelength of $532 \mathrm{~nm}$. It has been found that in comparison with the holes produced by laser drilling in air, the holes generated by laser drilling process in water has less spatters on the surface, but higher in-wall roughness. The etching rate achieved by laser drilling in water is lower than laser drilling in air at low pulse energies, but at high laser pulse energies, the etching rate can be three times higher than laser drilling in air. Garcia-Giron et al. (2016) have studied laser ablation of alumina, 8YSZ and glass-ceramic workpiece samples confined in water and in ethylene glycol using nanosecond laser pulses at $1064 \mathrm{~nm}$, and it has been found that the water-confined laser ablation can yield higher ablation yields for all the three types of samples, while under the confinement of ethylene glycol, machining can only be realized for the brittle glass-ceramic sample. 
Recently, a new machining process, called ultrasound-assisted water-confined laser micromachining (UWLM), was proposed by the corresponding author of the present paper $(\mathrm{Wu}$, 2014). In the proposed UWLM process, a laser beam is incident onto the front surface of a workpiece (that is, the surface facing the incoming laser beam) to remove materials from the workpiece to produce or modify micro-features, and in-situ ultrasonic waves are also applied to the laser ablation location (e.g., from above the workpiece front surface using an ultrasonically vibrating horn), which is immersed in water. The ultrasonic waves energize the water and generate cavitations, which may produce or enhance related beneficial effect(s) in water, such as cleaning, to decrease or minimize laser machining defect(s) (such as debris deposition that is often seen for laser ablation in air), and may also generate a much higher material removal rate than laser ablation in water without ultrasound. Other types of liquids may also be potentially used in UWLM besides water.

The first journal article on the experimental study of UWLM by the authors is Liu et al. (2014). It has been found by Liu et al. (2014) that under the investigated conditions, UWLM can yield much less debris deposition than laser machining in air, while it can produce much higher material removal rate per laser pulse than laser machining in water without ultrasound for copper workpieces. Liu et al. (2014) have also found that the enhanced material removal rate, which has been obtained in the studied UWLM process, cannot be achieved if a workpiece is lasermachined in water without ultrasound, and then after the machining process, the workpiece is treated by ultrasound in water using ultrasound conditions similar to those in the studied UWLM process.

Charee et al. (2016) reported research work on ultrasonic assisted laser machining of silicon in water, where the ultrasonic waves were applied by ultrasonically vibrating the bottom 
wall of the water container. Charee et al. (2016) have found that ultrasonic assisted laser machining of silicon in flowing water under suitable ultrasound conditions may yield an enhanced machining performance than general laser ablation in water. They attribute this to the flushing effect, etc., of the water energized by ultrasound. However, they did not report any direct in-situ process imaging evidence (during the machining process) for the underlying mechanism of the ultrasound-assisted laser machining process they studied. In addition, it should be noted that Charee et al. (2016) showed that under suitable ultrasound conditions the laser-ablated groove depth can be increased when ultrasound is applied, while they also found that under many other studied conditions, the laser-machined groove depth is shallower with ultrasound than that without ultrasound.

So far, an in-situ time-resolved observation of the UWLM process has been rarely reported, which is, however, very critical for understanding the process mechanism. Besides, an experimental study that investigates the UWLM process (that utilizes an ultrasonic horn placed above the workpiece front surface) on multiple types of metal workpieces (instead of just on one type of metal) has been rarely reported.

In this paper, experimental studies and time-resolved in-situ observations of the UWLM process have been performed. Different from the authors' previous work in Liu et al. (2014), which reported UWLM results on copper using 50 laser pulses, in this paper multiple types of metal workpieces, such as bronze, titanium, and stainless steel besides copper, have been studied, where $a$ variety of laser pulse numbers have been used. The ultrasonic waves are generated by a vibrating horn placed near the workpiece surface. The surface morphologies, profiles and depths of holes drilled on the metallic workpieces through UWLM have been characterized and compared with those drilled by laser ablation in water without ultrasound. As shown later in this 
paper, under the studied conditions using 15 to 200 laser pulses, the UWLM process has produced much higher material removal rate per pulse than laser ablation in water without ultrasound for all the different metals investigated. In addition, in-situ time-resolved shadowgraph imaging of the UWLM process has been performed in this study, which has revealed one important likely underlying mechanism of the investigated UWLM process for the greatly enhanced material removal rate, which will be discussed in more details later. In the authors' previous work in Liu et al. (2014), an ultrasonic horn vibrating at $20 \mathrm{kHz}$ has been applied in the UWLM process, and the workpiece surface is parallel to the lab ground (and hence the laser beam propagation direction is vertical to the lab ground). To demonstrate that the UWLM process can be performed under a different ultrasonic frequency and a different workpiece orientation, in this paper a $40 \mathrm{kHz}$ ultrasonic horn is used and the workpiece surface is vertical (and hence the laser beam is parallel) to the lab ground as shown later in Fig.1. However, please note that it is not the purpose of this paper to perform a comparative study of UWLM processes under different ultrasonic frequencies or workpiece orientations, which may be a good topic for future research work in this area.

It should be noted that the UWLM process is very different from the ultrasonic vibrationassisted laser machining without water confinement reported in the literature, and the latter does not involve the effect of ultrasound-energized water (which is, however, a critical component in the UWLM process). Lau et al. (1994) carried out a study of the drilling of aluminum-based metal matrix composites using 2-ms laser pulses, where the drilling process is assisted by ultrasonic vibration of the workpiece under an air jet at $20 \mathrm{kHz}$ (without any water confinement of the workpiece). It has been found in the study that the ultrasonic vibration assistance can increase the laser-drilled hole depth by up to $20 \%$, and can also result in less tapering. Zheng and 
Huang (2007) have performed an investigation of laser drilling of Nitinol samples using femtosecond laser pulses under the assistance of ultrasonic vibration of the workpiece sample at $40 \mathrm{kHz}$ without any water confinement, and it has been found that the application of ultrasonic vibration during laser drilling has enhanced the aspect ratio and the wall surface finish of laserdrilled microholes.

\section{Experiments}

Figure 1 shows the schematic diagram of the experimental setup for the UWLM process and the related time-resolved shadowgraph imaging observation. In this study, a Q-switched Nd:YAG laser (Spectra-Physics, Quanta-Ray INDI) is employed. The laser is coupled with a second-harmonic generator that gives a laser beam at a wavelength of $\sim 532 \mathrm{~nm}$ with a full-widthat-half-maximum (FWHM) pulse duration of around $7 \mathrm{~ns}$. The laser beam passes through a plano-convex lens (focal length, $\mathrm{f}=75 \mathrm{~mm}$ ) and irradiates the workpiece that is placed inside a water tank (the optics between the lens and the laser are not shown in Fig.1 for simplicity). The water tank has four walls made of uncoated borosilicate glass. The dimension of the tank is around $\sim 120 \mathrm{~mm} \times 120 \mathrm{~mm} \times 75 \mathrm{~mm}$, and is filled with water. The path length of the laser beam in water is about $50 \mathrm{~mm}$. The UWLM process on different types of metallic workpieces have been studied in this paper, which include 510 bronze, 101 copper, 304 stainless steel and titanium grade 2 sheets (purchased from McMaster-Carr). The purchased 304 stainless steel sheet comes with a mirror-like surface, and hence is used in the experiments without being further polished. All the other workpiece material sheets come with a rough surface, which are polished (using 2000-grit abrasive papers in the final polishing step) before they are used in the experiments. The incoming laser pulse energy to the tank is $\sim 2.3 \mathrm{~mJ}$, which is measured by 
placing an energy sensor (Coherent, J-25MT-10kHz) between the tank wall and the focusing lens before performing laser ablation on the workpiece. It has been found that the pulse energy is reasonably stable during the experiment.

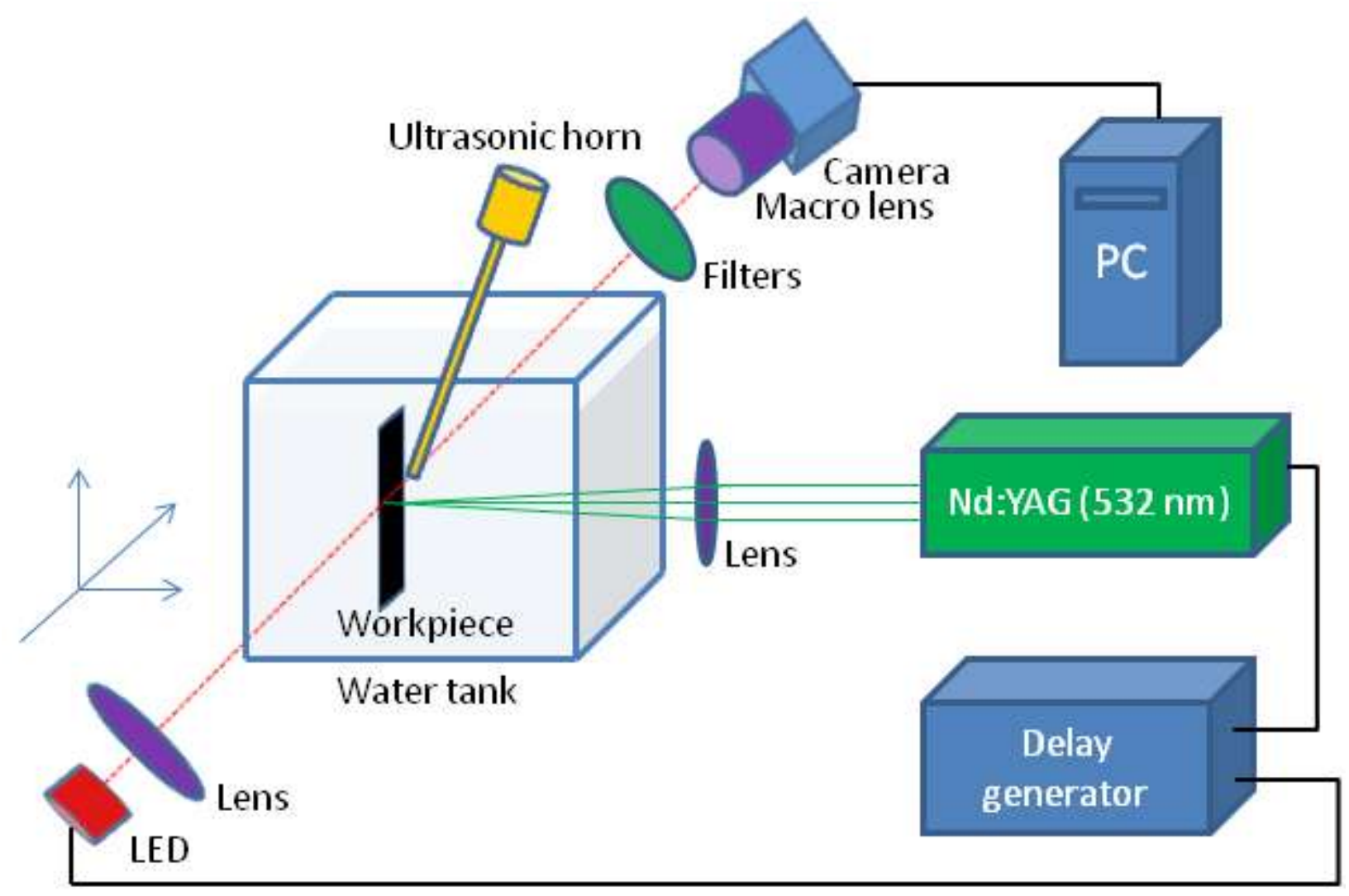

Figure 1. Schematic diagram of the experimental setup for the UWLM process in this study and the related shadowgraph imaging observation (not drawn to scale; not all components are shown).

The laser spot size on the workpiece surface immersed in water has been roughly estimated based on the laser-produced crater sizes on a titanium grade 2 sample and the ablation threshold of the material. Vladoiu et al. (2008) studied the ablation of metals in air using 4.5-ns laser pulses at the wavelength of $532 \mathrm{~nm}$, in which it has been found that the laser ablation threshold for titanium is around $\sim 1 \mathrm{~J} / \mathrm{cm}^{2}$. If it is roughly assumed that the ablation of titanium 
in water by the 532-nm nanosecond laser pulses used in this study has a similar ablation threshold fluence, then the laser spot radius on the workpiece surface in this study can be roughly estimated to be around $\sim 45 \mu \mathrm{m}$, based on the measured radii of craters on a titanium grade 2 sample ablated by 15 laser pulses in water without ultrasound and on the approximate assumption that the laser beam intensity and local fluence follow a Gaussian distribution on the workpiece surface.

During the UWLM process, in-situ ultrasonic waves are generated by an ultrasonic micro-tip horn, which has a tip diameter of $\sim 3.2 \mathrm{~mm}$ and is driven by an ultrasonic generator at $40 \mathrm{kHz}$ (Branson, SLPe). The ultrasonically vibrating horn tip is placed near the laser ablation location on the workpiece. During the UWLM process, the horn is operated at $10 \%$ of its full amplitude (which means the horn tip vibrates at a peak-to-peak amplitude of around $12 \mu \mathrm{m}$ according to the user manual: Sonifier ${ }^{\circledR}$ Cell Disruptor Model SLPe User's Manual EDP 100214-254 Rev. B by Branson Ultrasonics Corporation) throughout the period of laser ablation. As a comparison, experiments have also been performed on laser ablation in water without ultrasound. In this case, the horn is not vibrating, but is still kept at the same location as that in the UWLM process.

Different numbers of laser pulses, 15, 30, 60, 100 and 200, have been used for laser ablation in water with ultrasound (UWLM) and for laser ablation in water without ultrasound on the four different types of workpiece materials. The employed laser pulse repetition rate is $2 \mathrm{~Hz}$. To handle uncertainties in the experimental system, typically four ablations have been performed under each experimental condition, and also the UWLM process and laser ablation in water without ultrasound are performed alternatively on the workpiece. The surface profiles and hence ablation depths of laser ablated workpieces are characterized using a confocal microscopy 
system (Zeiss, LSM 710) that has an Argon laser (488 nm) illumination source. For each experimental condition, typically four ablations are performed, and the corresponding four depths are measured using the confocal microscope, the average of which gives the ablation depth under the experimental condition. The surface morphologies of laser ablated workpieces are observed with a scanning electron microscope (SEM) (JEOL, JSM-T330).

Figure 1 also includes the major components for time-resolved shadowgraph imaging of the laser ablation process in water with ultrasound (UWLM) and without ultrasound. The illumination source for the imaging is a high brightness red LED (light-emitting diode), which is driven by a pulsed current supply (not drawn). The LED light passes through the collimating lenses (which is to reduce its divergence), and then illuminates the region of interest (the region near the laser ablated workpiece surface location), and forms a shadowgraph image on the detector of a CMOS camera (Point Grey, BFLY-U3-23S6M-C, 1920 x 1200 pixels). A macro lens (Canon, MP-E 65mm) is coupled with the camera to produce a spatial magnification, The focus of the macro lens is adjusted to be at the region above the laser ablated workpiece surface location (here, "above" means the direction normal to the workpiece surface and pointing out of the workpiece). The LED light is in a pulsed mode with FWHM durations of $\sim 200$ to $\sim 400 \mathrm{~ns}$ used in this work. The relative timing of the laser pulse and the LED illumination light pulse is controlled through a digital delay generator (Berkeley Nucleonics, Model 577). In the experiment, one image is taken after one single laser pulse, where the delay time of the LED light pulse after the laser pulse is varied from $\sim 50 \mu$ s to $\sim 500 \mathrm{~ms}$. In this way, shadowgraph images at different times can be taken through multiple experiments. During the experiment, the CMOS camera is powered on and its exposure is also controlled using the delay generator (connection line not drawn in Fig.1). A notch filter (Thorlabs, NF533-17) and a bandpass filter 
(Omega Optical, 625BP70, center wavelength: 625nm, FWHM bandwidth: $70 \mathrm{~nm}$ ) are placed in front of the macro lens to block most of the scattered laser light (if any) at $\sim 532 \mathrm{~nm}$ and to block most of the un-wanted light that is outside the bandpass filter's narrow passing wavelength range, in order to facilitate the shadowgraph image formation on the camera detector.

\section{Results and discussions}

3.1 Surface profile and ablation depth

Figure 2 shows the two-dimensional (2D) surface profiles of holes on a copper workpiece drilled by laser ablation in water with ultrasound (that is, by the UWLM process) in the left column and by laser ablation in water without ultrasound in the right column. The profiles are measured using a confocal microscope. Figure 2(a) to (e) shows the holes drilled by 15, 30, 60, 100 and 200 pulses, respectively.

It can be seen from the figure that the hole depth increases as the pulse number increases from 15 to 200 for both the UWLM process and laser ablation in water without ultrasound. However, when laser pulse number is the same, the hole depth produced by the UWLM process is significantly larger than that produced by laser ablation in water without ultrasound. Under the same laser pulse number, the hole produced by UWLM also has a smaller taper angle and steeper side walls. The images in Fig.2 have clearly shown a significantly much higher material removal rate for the UWLM process than laser ablation in water without ultrasound under the investigated conditions. 


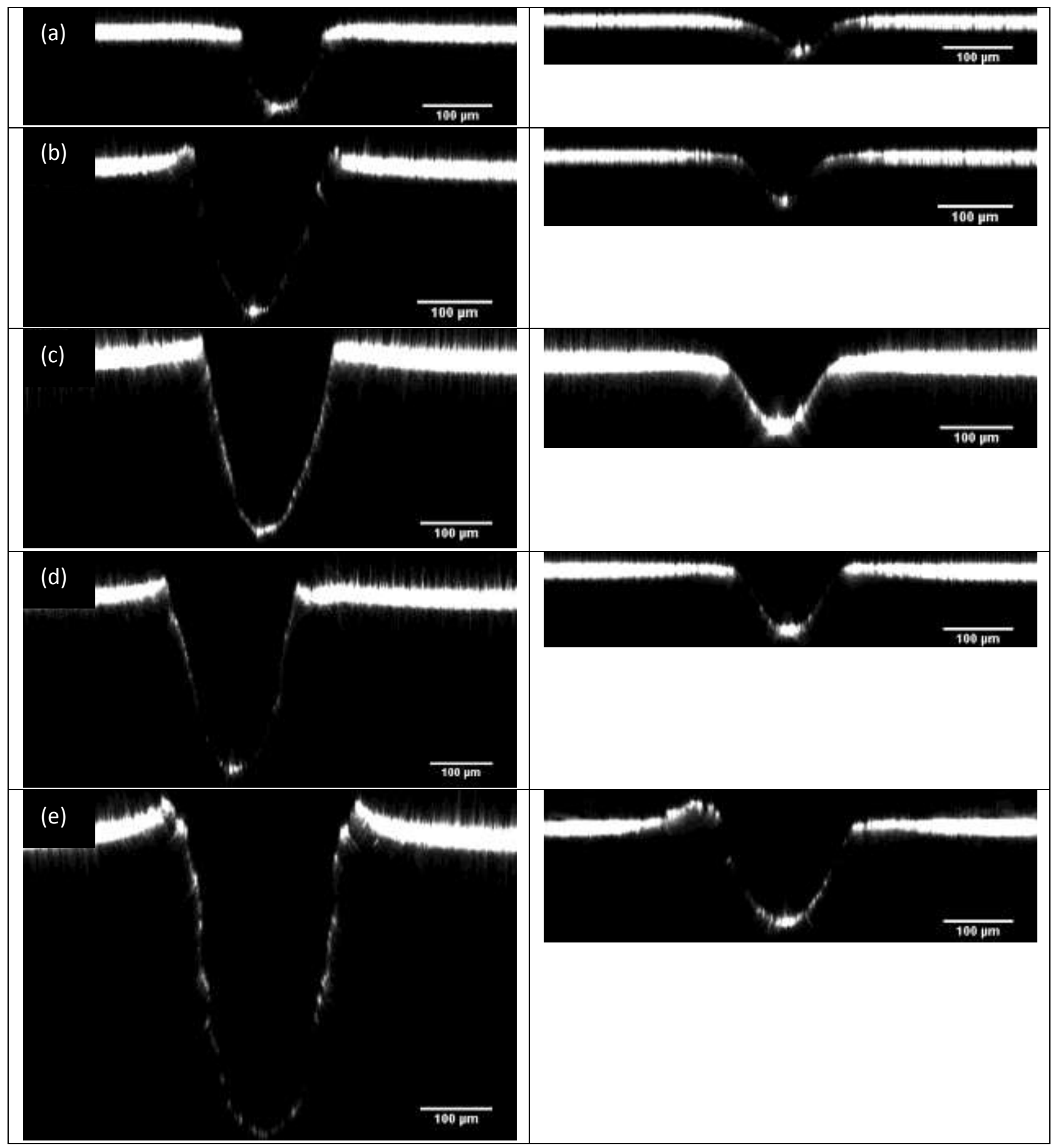

Figure 2. Measured two-dimensional (2D) depth profiles of the holes drilled on copper through the UWLM process (i.e., laser ablation in water with ultrasound) (left) and through laser ablation in water without ultrasound (right) using 15 (a), 30 (b), 60 (c), 100 (d) and 200 (e) laser pulses (the electronic version of the figure may look more clear than its printed version). 
The hole drilled by UWLM in Fig. 2e is much deeper than all the other holes shown in the figure. This corresponds to a much larger amount of material removal, and a ridge can be observed around the hole boundary in Fig. 2e, which is expected to be mainly due to one or a combination of workpiece mechanical deformation and laser-induced workpiece melt resolidification, during the removal of such a large amount of materials.

Figure 3 shows measured 2D profiles of holes drilled on bronze, stainless steel and titanium workpieces using 100 laser pulses through UWLM (the left column) and through laser ablation in water without ultrasound (the right column). For all the three types of workpiece materials, the holes drilled by UWLM have larger depths and smaller taper angles than those drilled by laser ablation in water without ultrasound. Figure 3 shows again that under the investigated conditions, UWLM has much higher material removal rates than laser ablation in water without ultrasound for the three different types of metal workpiece materials. It should be noted that for Figs. 2 and 3 even through a high gain of the detector of the confocal microscope is used during the profile measurement, the data for some points of the hole side and bottom walls is still missing, which is expected to be due to the relatively low reflectivity of the relevant surface. The high detector gain has led to the fuzzy profile of the un-ablated workpiece surface surrounding the hole, which is expected to be due to the relatively high reflectivity of the polished un-ablated workpiece surface. The hole drilled by UWLM in Fig. 3a corresponds to a much larger amount of material removal than all the holes produced by laser ablation in water without ultrasound in this figure. Similar to Fig. 2e, a ridge is observable around the hole boundary in Fig. 3a, which is expected to be mainly due to similar reasons mentioned earlier for Fig. 2e. 


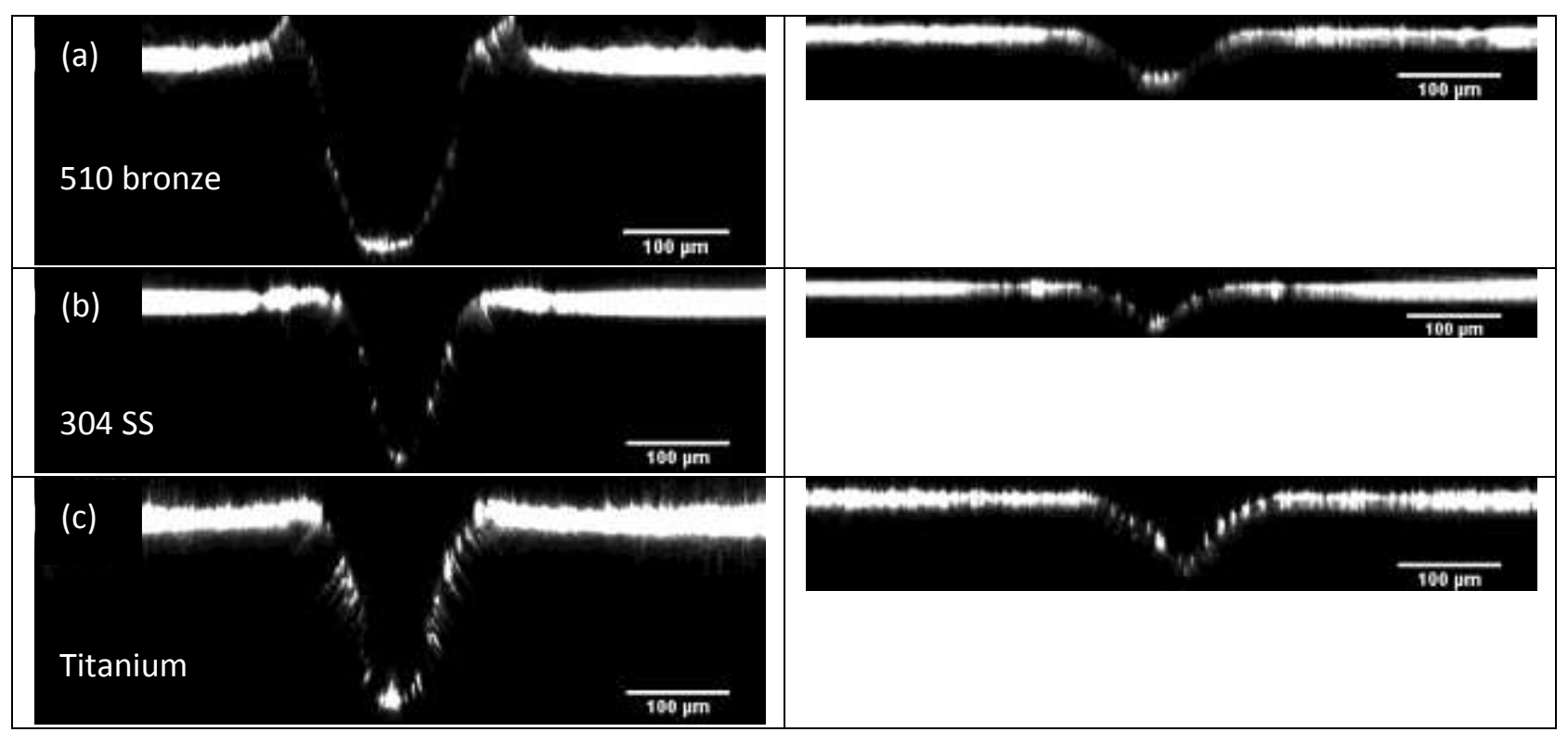

Figure 3. Measured 2D depth profiles of the holes drilled on (a) bronze, (b) stainless steel (SS) and (c) titanium workpieces through the UWLM process (left) and through laser ablation in water without ultrasound (right) using 100 laser pulses (the electronic version of the figure may look more clear than its printed version).

Figure $4 \mathrm{a}$ to $4 \mathrm{~d}$ shows the ablation depth vs laser pulse number for holes drilled on copper, bronze, stainless steel and titanium workpieces, respectively. The depths are measured using a confocal microscope. For the results on copper workpieces shown in Fig. 4(a), laser ablation in water without ultrasound using 15 laser pulses has yielded an ablation depth (i.e., hole depth) of $\sim 42 \mu \mathrm{m}$, while the depth produced by UWLM using 15 pulses is $\sim 104 \mu \mathrm{m}$. The difference in the ablation depths produced by UWLM and by laser ablation in water without ultrasound increases as the laser pulse number increases in the range shown in Fig.4a. When the laser pulse number is 200 , the ablation depth produced by UWLM is $\sim 397 \mu \mathrm{m}$, which is more than 3 times the depth produced by laser ablation in water without ultrasound, which is $\sim 129 \mu \mathrm{m}$. For bronze, stainless steel and titanium workpieces, Fig.4b to $4 \mathrm{~d}$ shows that the ablation depths produced by UWLM are still much larger than those produced by laser ablation in water without 
ultrasound under the same laser pulse number. When laser pulse number is 200 , the depths of holes drilled by UWLM are $\sim 273 \%, \sim 383 \%$ and $\sim 234 \%$ of those produced by laser ablation in water without ultrasound for bronze, stainless steel and titanium workpieces, respectively.
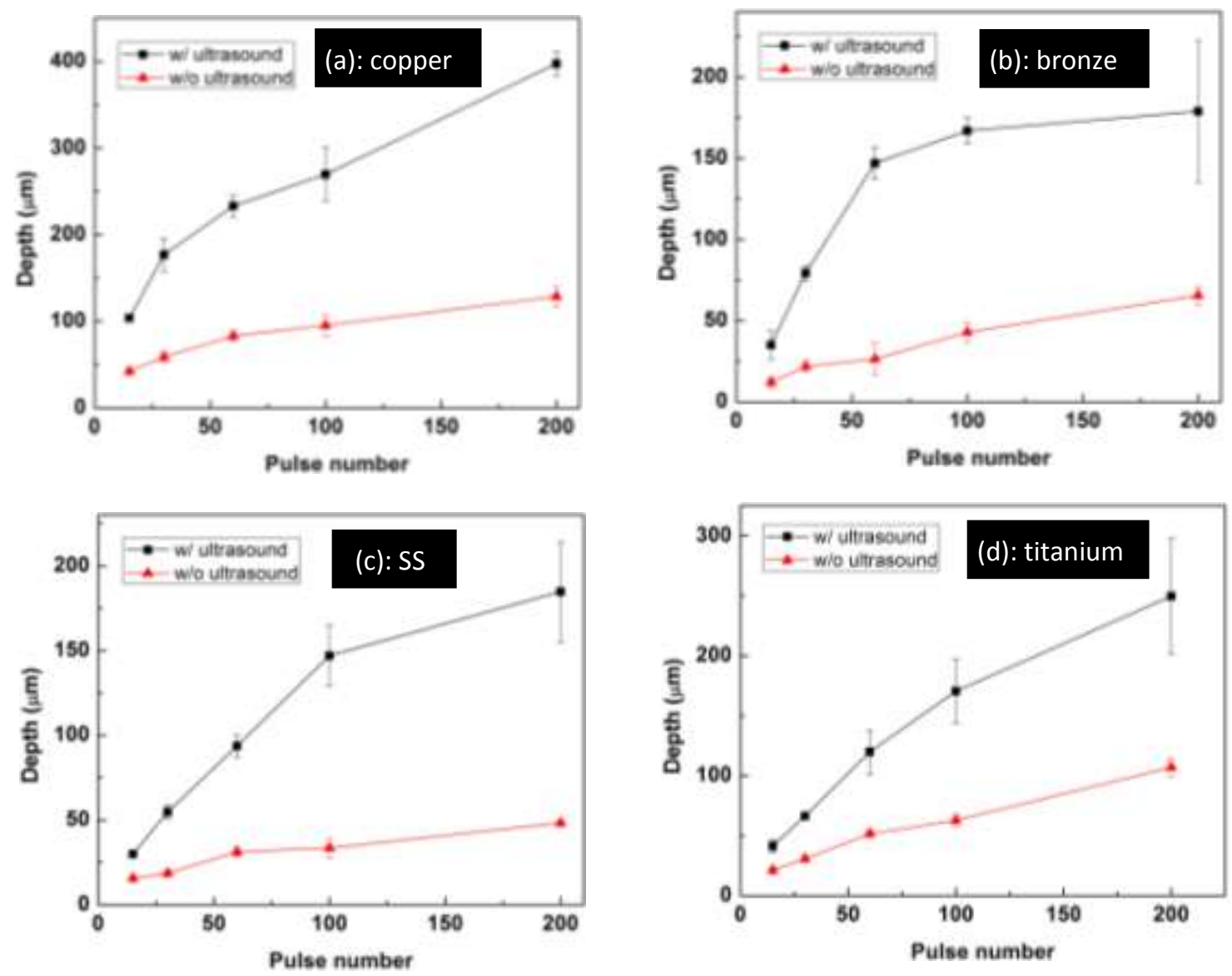

Figure 4. The depths of holes drilled on (a) copper, (b) bronze, (c) stainless steel (SS), and (d) titanium workpieces vs. laser pulse number, through the UWLM process (i.e., laser ablation in water with ultrasound) and through laser ablation in water without ultrasound.

\subsection{SEM images}

Figure 5 shows the SEM images of holes on copper workpieces drilled by the UWLM process (the left column) and by laser ablation in water without ultrasound (the right column), using $15,30,60,100$ and 200 pulses. 


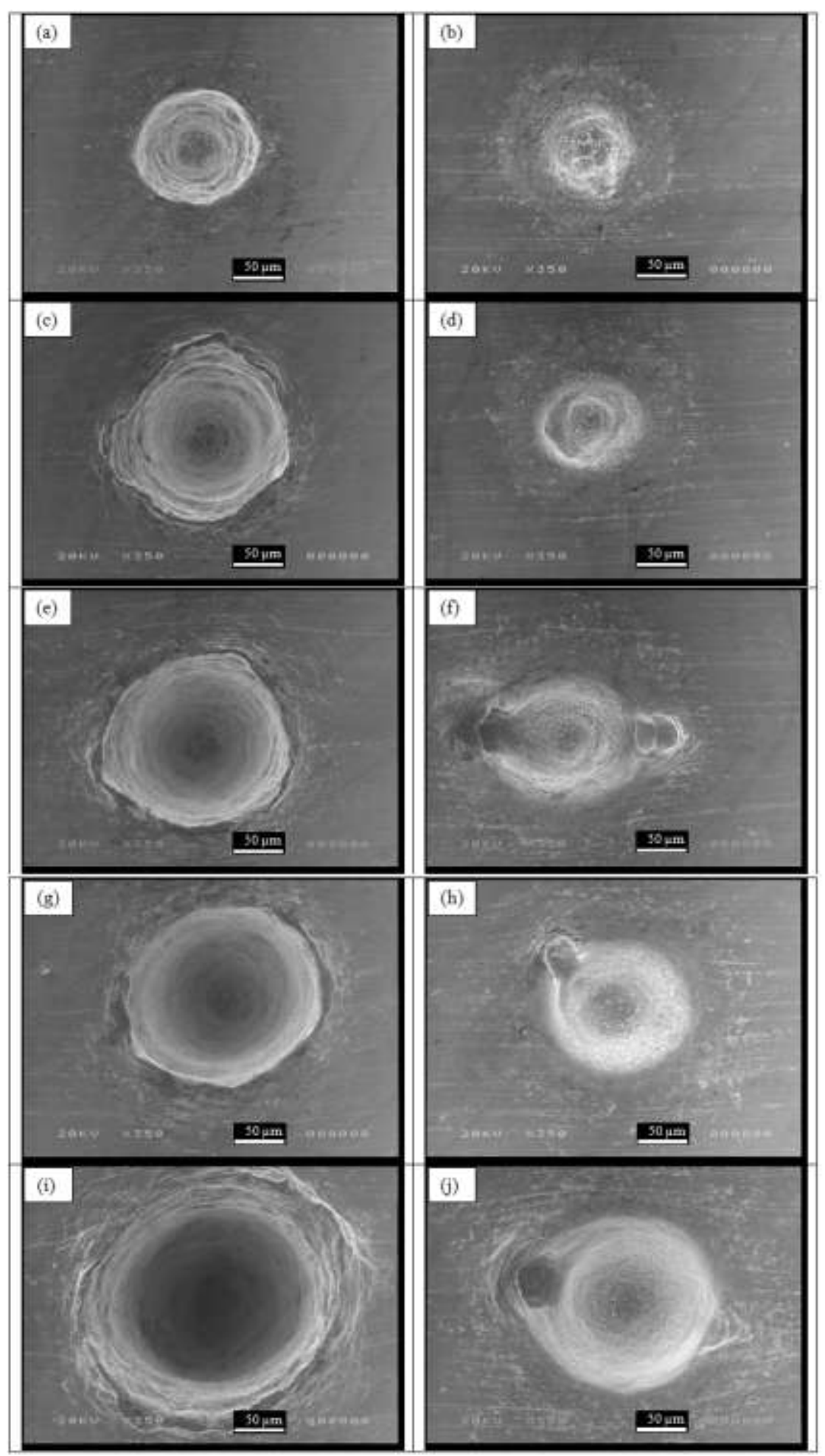

Figure 5. SEM images of the holes drilled on copper through the UWLM process (left) and through laser ablation in water without ultrasound (right) using 15 (a, b), 30 (c, d), 60 (e, f), 100 $(\mathrm{g}, \mathrm{h})$, and $200(\mathrm{i}, \mathrm{j})$ laser pulses. 
In both columns of Fig. 5, the hole depths and diameters at 200 pulses are obviously larger than those at 15 pulses. However, the hole morphologies produced by UWLM and by laser ablation in water without ultrasound look very different in the SEM images. Under the same laser pulse numbers, the holes produced by UWLM appear much deeper with steeper sidewalls than those produced by laser ablation in water without ultrasound, which agrees with the profile measurement results by the confocal microscope shown in Fig. 2. On the other hand, the holes produced by laser ablation in water without ultrasound look much shallower.

As introduced earlier, during the experiments of laser ablation in water without ultrasound, the ultrasonic horn is still placed nearby (which is not vibrating), so that the only major difference from a UWLM process is that the horn is vibrating ultrasonically in the latter process. In this way, the differences in the machining results can be mainly attributed to the applied ultrasonic waves. For the holes produced by laser ablation in water without ultrasound shown in Fig. 5(f), (h) and (j), small craters can be observed near the holes, and a hypothesized explanation for the formation of such craters is that they are formed probably due to the pressure waves that are generated during the laser ablation process and then reflected by the non-vibrating horn placed nearby back to the soft copper surface (further work is still needed to verify this hypothesized explanation). The pressure waves may be generated due to the high-pressure plasma induced by laser ablation in water at around the workpiece surface, and/or the evolution of cavitation bubble(s) in water resulted from laser ablation (For example, Montross et al. (2002) have reviewed the laser shock peening process, and the review has shown that the pressure of the plasma induced by laser ablation of a solid in water during the laser shock peening process can often have a high pressure of a few GPa. Chen et al. (2004) have studied laser ablation of a metal in water, in which it was shown that both laser-induced plasma and the collapse of laser-induced 
cavitation bubble can generate shock waves in water.). Such small craters are not observed if the horn is removed. Such craters are also not observed near the holes produced by the UWLM process shown in Fig. 5. The reason is likely to be that the horn is vibrating during the UWLM process, which may generate cavitation bubbles near the horn surface. This may lead to a more scattered reflection of the pressure wave towards different directions, and hence the wave reflected back to the copper surface near the hole is not strong enough to generate a crater. Future work is still needed to fully understand this.

Figure 6 shows the SEM images of the holes drilled on bronze, stainless steel, and titanium workpieces through the UWLM process (the left column) and through laser ablation in water without ultrasound (the right column) using 100 laser pulses. Again, the holes produced by UWLM for all the three types of metal workpieces appear much deeper with steeper side walls than those produced by laser ablation in water without ultrasound. The small craters, as in Fig. 5f, h and $\mathrm{j}$, are not observed in Fig. 6. This is likely to be due to the higher hardness of the workpieces in Fig. 6 than copper (based on the product information from McMaster-Carr), and hence the pressure wave cannot generate a crater. 


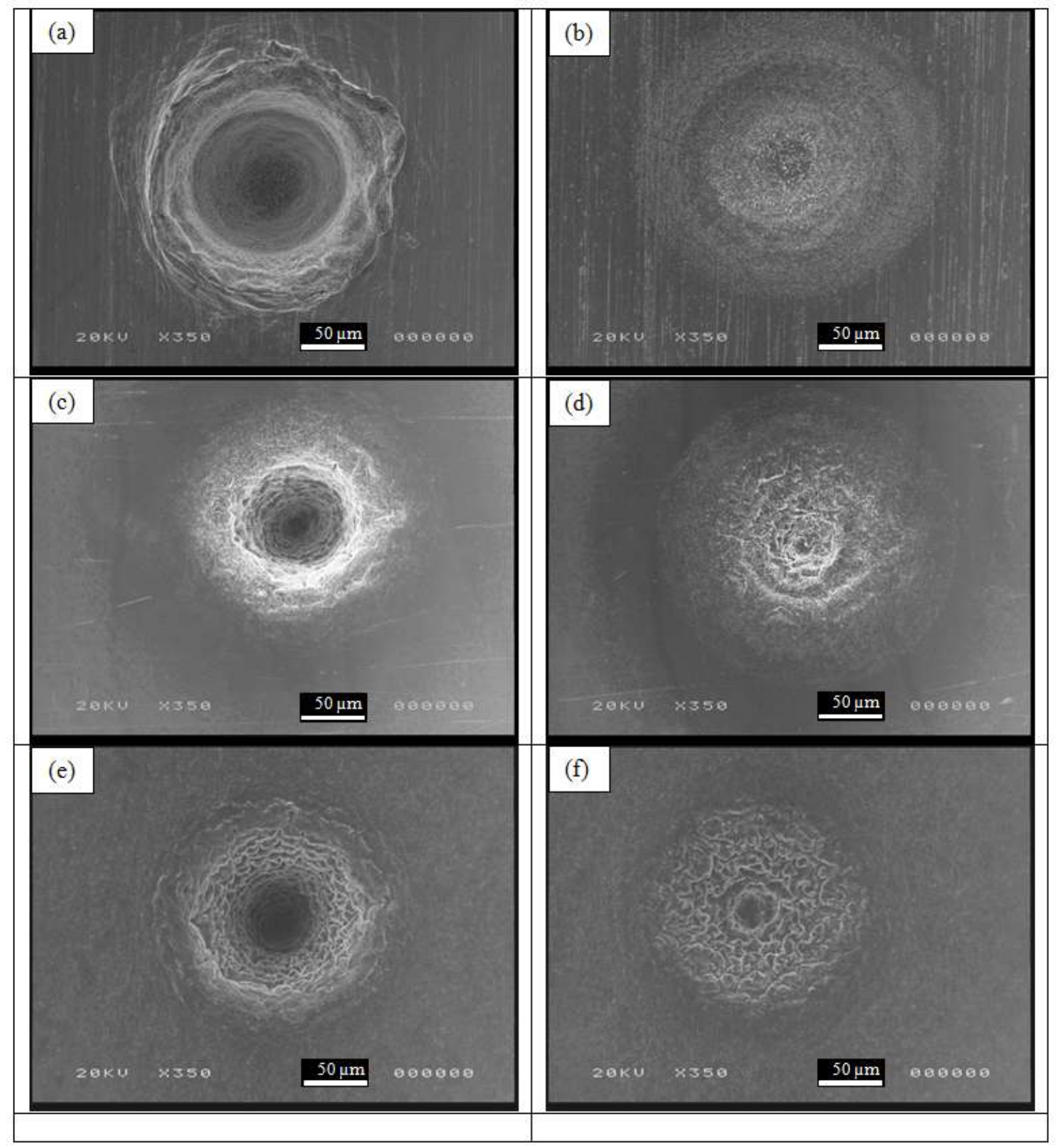

Figure 6. SEM images of the holes drilled on bronze (a,b), 304 stainless steel (c,d), and titanium $(\mathrm{e}, \mathrm{f})$ workpieces through the UWLM process (left) and through laser ablation in water without ultrasound (right) using 100 laser pulses. 


\subsection{In-situ shadowgraph imaging}

Figure 7 left and right columns show shadowgraph images for the laser ablation process in water with ultrasound (i.e., the UWLM process) and laser ablation in water without ultrasound, respectively. The images are taken at delay times of $\sim 50 \mu$ s to $\sim 500 \mathrm{~ms}$ after the shot of the corresponding laser pulse. After a high-intensity nanosecond (ns) laser pulse irradiates a metal workpiece surface immersed in water, a plasma plume at the workpiece surface may be produced, which evolves with time, and may eventually lead to a cavitation bubble in the ambient water. Figure 7 (a) and (b) show the shadowgraph images taken at a delay time of $\mathrm{t}=$ $\sim 50 \mu$ s for laser ablation with ultrasound (i.e., the UWLM process) and without ultrasound, respectively. The images clearly show a bubble in a near hemispherical shape right above the workpiece surface, whose center is located at the laser ablation location.

In Fig.7a, the vertical dashed line indicates the approximate workpiece surface location, which is at the same corresponding location for all the other images in Fig. 7. The polished workpiece surface has a high reflectivity and a mirror image is formed on the right side of the dashed line for objects near the workpiece surface. At the delay time of $t=\sim 50 \mu \mathrm{s}$, the bubble shape and size produced by laser ablation in water with and without ultrasound are similar. At $\mathrm{t}=$ $\sim 130 \mu \mathrm{s}$, the bubbles produced under both laser ablation conditions reach their approximate maximum size as shown in Fig. 7(c) and (d), where the bubbles still have a near hemispherical shape. In Fig.7(a) to (d), a bright spot near the bubble center can be seen. This kind of bright spot is also observed in the shadowgraph images given in Lazic et al. (2012) for a bubble produced by laser ablation in water, which is expected to be mainly due to multiple reflections of the illumination light at the bubble wall as mentioned by Lazic et al. (2012). 

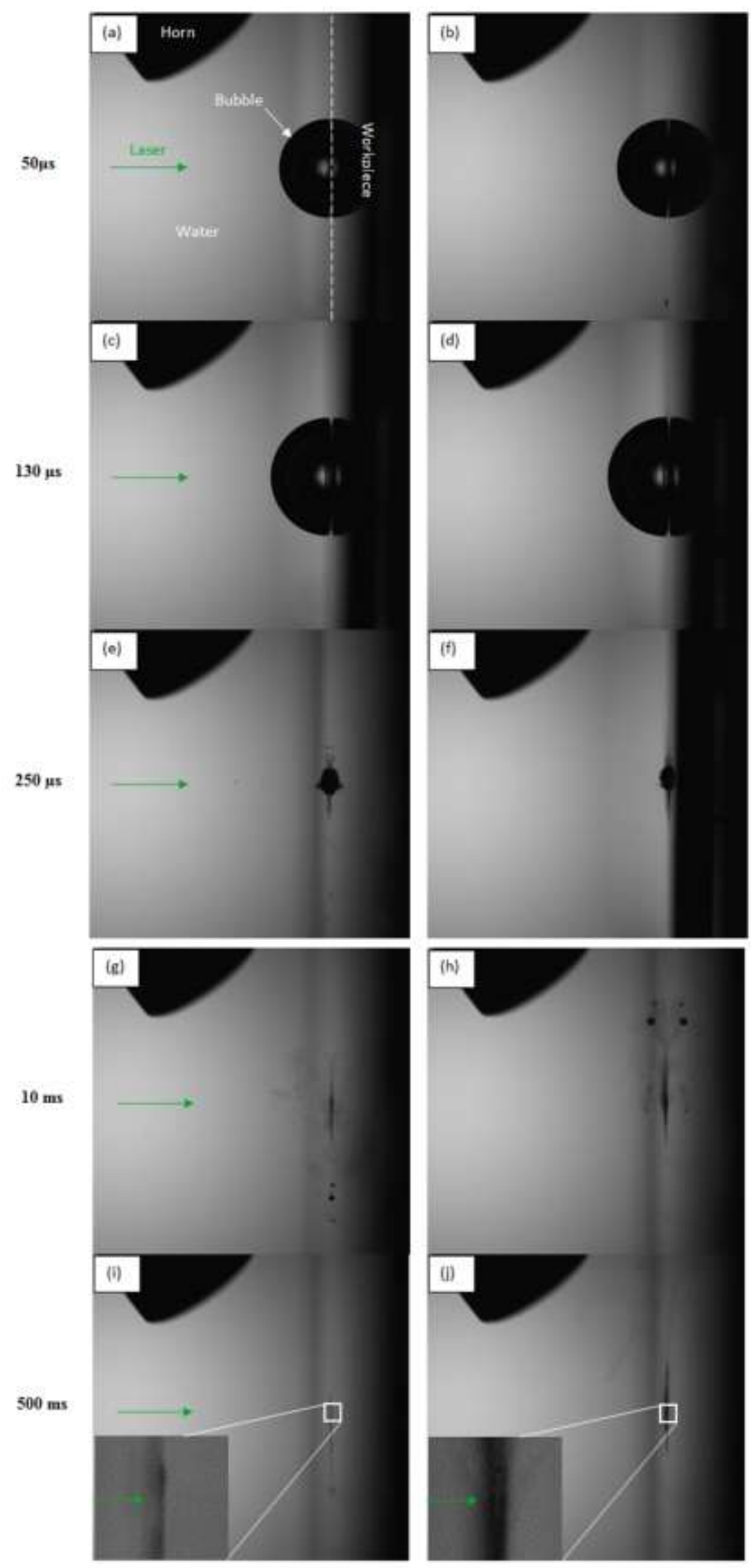
Figure 7. Shadowgraph images of the area around a laser ablation site at different delay times after a corresponding laser pulse for the UWLM process (left column) and for laser ablation in water without ultrasound (right column). For each image, the corresponding physical domain size is $\sim 7.3 \mathrm{~mm} \times 7.0 \mathrm{~mm}$. The surface of the bronze workpiece is indicated by the vertical dashed line drawn in Fig. 7 (a), and the laser beam is schematically and approximately indicated by a horizontal arrow in some images (please note the corresponding laser pulse ended long before the moments in these images). The insets in Fig. 7(i) and 7(j) give a further magnified view of the area very near the laser ablation site, showing that the material cloud appears thicker in Fig.7(j) than (i) (the electronic version of the figure may look more clear than its printed version).

After the bubbles reach their maximum size, they will shrink, and also their shape will gradually deviate from the previous near hemispheric shape. At $t=\sim 250 \mu$ s, as shown Fig. 7(e) and (f), the bubbles under both laser ablation conditions have collapsed, which has led to a cloud that is expected to include smaller bubbles, and the bubbles may undergo several rebounds. So far, there is no significant difference observed in the shadowgraph images for laser ablation in water with ultrasound (i.e., the UWLM process) and the images for laser ablation in water without ultrasound.

However, at a delay time of $\mathrm{t}=\sim 10 \mathrm{~ms}$, a small cloud of material near the laser ablated workpiece surface location can be observed in the image in Fig $7(\mathrm{~g})$ for laser ablation in water with ultrasound (i.e., the UWLM process). The cloud of material appears like a faint haze in the water region nearby, and looks darker in the region at around the workpiece surface. It is expected that the cloud of material is composed of small bubbles and/or materials coming from the workpiece surface. Several larger bubbles can be seen in the region a little bit below the laser ablation site, which are expected to be bubbles pushed away due to the effects of the ultrasonic waves generated by the horn. The effects likely include the acoustic radiation force and/or the acoustic streaming resulted from the ultrasonically vibrating horn. Torr (1984) explained the 
physical origin of the acoustic radiation force, and it has been demonstrated that the contributing sources to the radiation force may include the time-averaged sound pressure that is not equal to zero and the beam-transferred momentum. Nowak et al. (2015) studied the acoustic streaming and the translation of bubbles induced by an ultrasonic horn at $20 \mathrm{kHz}$ that can generate cavitations, in which it has been found that due to the presence of cavitation the liquid streaming velocities can be enhanced on average by a factor of 30 in comparison with the situation when cavitation is not present. Figure 7(h) shows the image for laser ablation in water without ultrasound at $\mathrm{t}=\sim 10 \mathrm{~ms}$, where the material cloud in the region at around the workpiece surface looks thicker than that in Fig 7(g) for the UWLM process.

Figure 7(i) and (j) show the images at $\mathrm{t}=\sim 500 \mathrm{~ms}$ for the UWLM process and for laser ablation in water without ultrasound, respectively. In Fig. 7(j), a relatively thick material cloud still exists near the laser-ablated workpiece surface location. However, in Fig.7(i), the material cloud appears much lighter, which is expected to be due to the more effective cleaning effect of the water energized by the in-situ ultrasonic waves from the horn during the UWLM process than the water without any ultrasound applied. In other words, some material in the cloud is driven away as a result of the ultrasonic waves from the horn, probably due to the acoustic radiation force and/or the acoustic streaming resulted from the ultrasonic waves.

During the laser ablation processes corresponding to Figs. 2 to $6, \mathrm{t}=500 \mathrm{~ms}$ is the time when the next laser pulse comes. For laser ablation in water without ultrasound, it is expected that the thick material cloud that is still at around the workpiece surface (as can be observed in. Fig.7(j)) will negatively affect the efficient coupling of the laser pulse energy to the workpiece and may decrease the laser intensity reaching the workpiece surface (through possible mechanisms, such as reflection and refraction at the bubble wall, scattering and/or absorption of 
laser pulse energy by the bubbles and workpiece material particles in the cloud. For example, Lazic et al. (2012) have found that the bubbles induced by laser ablation in water may cause beam defocusing and disturbance of the subsequent laser pulse). This may greatly reduce the material removal rate induced by the subsequent laser pulse(s). On the other hand, for laser ablation in water with ultrasound (i.e., the UWLM process), the in-situ ultrasonic wave from the horn can energize the water and enhance the water cleaning effect, which can make the material cloud at around the workpiece surface much lighter (as observed in Fig.7(i)). It is expected that this may significantly improve the coupling of the subsequent laser pulse(s)' energy to the workpiece, which then enhances the material removal rate. The above revealed mechanism is consistent with the authors' findings under the studied conditions in Fig.4 that the ablation depth difference between UWLM and laser ablation in water without ultrasound is typically larger as the laser pulse number increases. It should be noted that Fig.7 shows the imaging results for bronze, and similar imaging results have also been obtained for copper, stainless steel and titanium workpieces in this study, which are shown in Fig. 8.

It should be noted that each image in Figs. 7 and 8 is a representative one selected from multiple shadowgraph images taken during multiple laser ablation experiments at about the same delay time after the first laser pulse in each experiment (it should be noted that the "first" pulse in each experiment may not be ablating a fresh workpiece surface location, at which a certain number of laser pulses may have already been fired during previous experiments). 


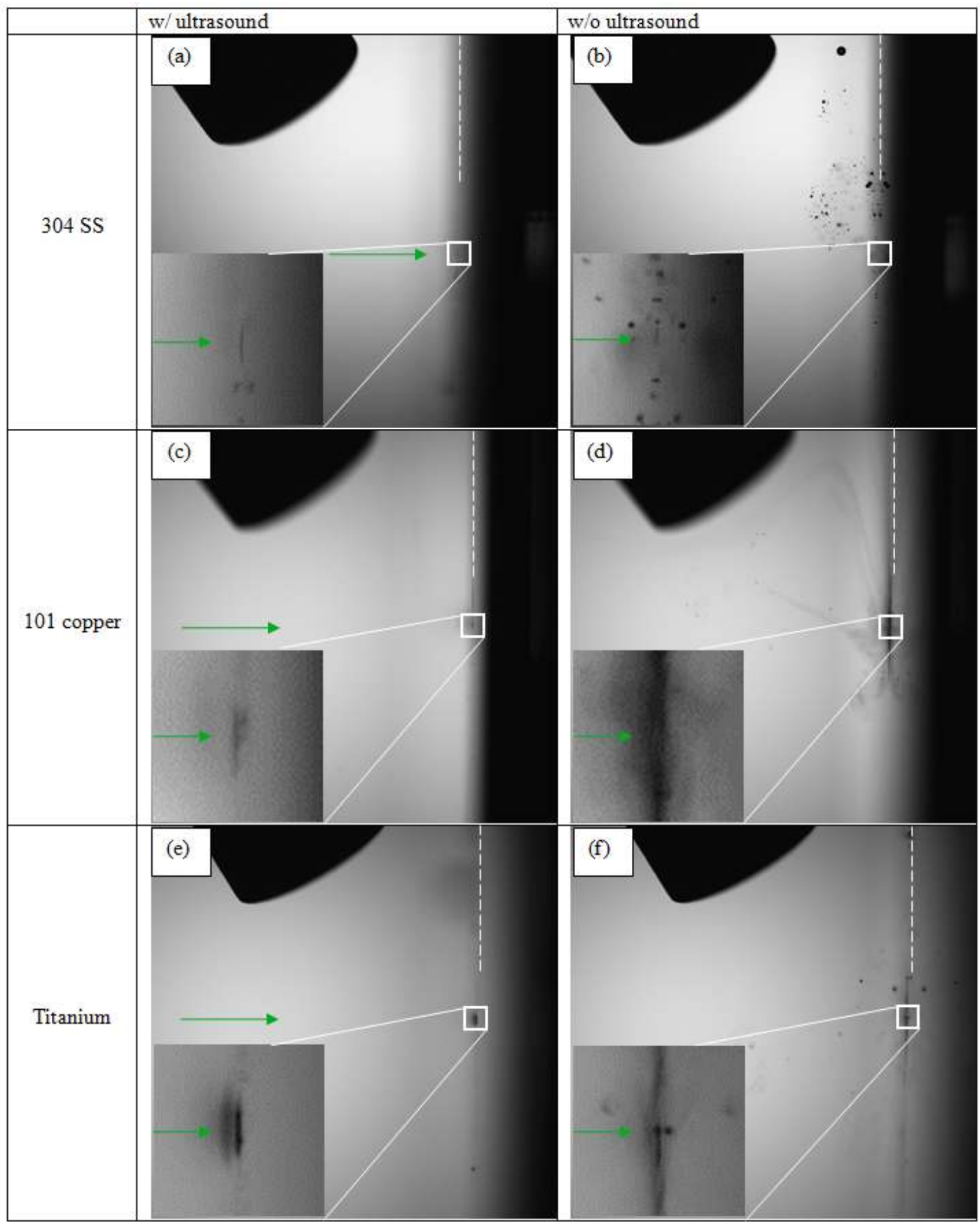

Figure 8. Shadowgraph images of the area around laser ablation sites on different workpieces at $\sim 500 \mathrm{~ms}$ after a corresponding laser pulse for the UWLM process (left column) and for laser ablation in water without ultrasound (right column). The corresponding workpieces are: 304 stainless steel ((a) and (b)), 101 copper ((c) and (d)), and titanium ((e) and (f)). For each image, the corresponding physical domain size is $\sim 7.3 \mathrm{~mm} \times 7.0 \mathrm{~mm}$. The approximate location of each workpiece surface is indicated by a vertical dashed line, and the laser beam is schematically and approximately indicated by a horizontal arrow (please note the corresponding laser pulse ended long before the moments in these images. The electronic version of the figure may look more clear than its printed version). 
In summary, the shadowgraph images in Figs. 7 (particularly Fig.7(i) and (j)) and 8 have revealed one important likely underlying mechanism for the much higher material removal rate of UWLM than laser ablation in water without ultrasound observed in this study for laser ablation using multiple (15 to 200) laser pulses. That is, the material cloud induced by laser ablation in water without ultrasound (which may consist of small bubbles and material particles coming from the workpiece surface) may linger at around the workpiece surface for a relatively long time, which may affect the coupling of the subsequent laser pulse(s)' energy to the workpiece, and hence reduce material removal rate. On the other hand, the in-situ ultrasonic wave in the UWLM process can energize the water, enhance the water cleaning effect, and reduce the amount of lingering material near the laser ablation site, which can enhance the energy coupling of the subsequent laser pulse(s), and therefore enhance the material removal rate. It should be noted that the above revealed mechanism is not necessarily the only mechanism involved. In addition, the involved mechanism(s) may also depend on laser, ultrasound and other parameters in the UWLM process. Therefore, extensive research work in the future is still needed to study the UWLM process.

\section{Conclusions}

The ultrasound-assisted water-confined laser micromachining (UWLM) process has been studied on copper, bronze, stainless steel and titanium workpieces, where ultrasonic waves are applied through a horn placed near the laser ablation location on the workpiece front surface. Under the investigated conditions using 15 to 200 laser pulses, it has been found that UWLM can yield much higher material removal rates than laser ablation in water without ultrasound. One 
important likely underlying mechanism for the enhanced material removal rate has been revealed through in-situ time-resolved shadowgraph imaging, which is, the in-situ ultrasonic wave during UWLM can enhance the water cleaning effect, and reduce the material cloud resulted from the previous laser pulse ablation that lingers at around the workpiece surface, which enhances the coupling of the subsequent laser pulse(s)' energy to the workpiece and hence increases the material removal rate.

This study has focused on the UWLM process at a low laser pulse frequency $(2 \mathrm{~Hz})$. It is certainly a good future research topic to investigate the UWLM process at much higher laser pulse frequencies. In particular, a UWLM process based on intense and short ultrasound pulses with controlled timing relative to high-frequency laser pulses will be a very interesting process to study.

\section{Acknowledgement}

This material is based upon work supported by the National Science Foundation under Grant Nos. CMMI 1543865 and CMMI 1055805. The authors also would like to thank Zhen Yang for his relevant help during the experimental work. 


\section{List of References}

Charee, W., Tangwarodomnukun, V., Dumkum, C., 2016. Ultrasonic-assisted underwater laser micromachining of silicon. Journal of Materials Processing Technology. 231, 209-220.

Chen, X., Xu, R.Q., Chen, J.P., Shen, Z.H., Jian, L., Ni, X.W., 2004. Shock-wave propagation and cavitation bubble oscillation by Nd: YAG laser ablation of a metal in water. Applied Optics. $43,3251-3257$.

Chien, W.T., Hou, S.C., 2007. Investigating the recast layer formed during the laser trepan drilling of Inconel 718 using the Taguchi method. The International Journal of Advanced Manufacturing Technology. 33, 308-316.

Dahotre, N. B., Harimkar, S., 2008. Laser Fabrication and Machining of Materials. Springer Science \& Business Media, New York.

Ehmann, K.F., 2007. A synopsis of U.S. micro-manufacturing research and development activities and trends. Proceeding of 4M2007 Conference on Multi-Material Micro Manufacture.

Garcia-Giron, A., Sola, D., Pena J.I., 2016. Liquid-assisted laser ablation of advanced ceramics and glass-ceramic materials. Applied Surface Science. 363, 548-554.

Kruusing, A., 2004. Underwater and water-assisted laser processing: Part 2-Etching, cutting and rarely used methods. Optics and Lasers in Engineering. 41, 329-352.

Lau, W.S., Yue, T.M., Wang, M., 1994. Ultrasonic-aided laser drilling of aluminium-based metal matrix composites. Annals of the CIRP. 43, 177-180.

Lazic, V., Jovicevic, S., Carpanese, M., 2012. Laser induced bubbles inside liquids: Transient optical properties and effects on a beam propagation. Applied Physics Letters. 101, 054101.

Liu, Z., Gao, Y., Wu, B., Shen, N., Ding, H., 2014. Ultrasound-assisted water-confined laser micromachining: A novel machining process. Manufacturing Letters. 2, 87-90.

Lv, J., Dong, X., Wang, K., Duan, W., Fan, Z., Mei, X., 2016. Study on process and mechanism of laser drilling in water and air. The International Journal of Advanced Manufacturing Technology. 86, 1443-1451.

Masuzawa, T., 2000. State of the art of micromachining. CIRP Annals - Manufacturing Technology. 49, 473-488.

Matsumura, T., Kazama, A., Yagi T., 2005. Generation of debris in the femtosecond laser machining of a silicon substrate. Applied Physics A: Materials Science \& Processing. 81, 13931398.

Montross, C.S., Wei, T., Ye, L., Clark, G., Mai, Y.W., 2002. Laser shock processing and its effects on microstructure and properties of metal alloys: a review. International Journal of Fatigue. 24, 1021-1036.

Morita, N., Ishida, S., Fujimori, Y., Ishikawa, K., 1988. Pulsed laser processing of ceramics in water. Applied Physics Letters. 52, 1965-1966.

Nowak, T., Cairós, C., Batyrshin, E., Mettin, R., 2015. Acoustic streaming and bubble translation at a cavitating ultrasonic horn. AIP Conference Proceedings. 1685, 020002. 
Tangwarodomnukun, V., Wang, J., Mathew, P., 2010. A Comparison of Dry and Underwater Laser Micromachining of Silicon Substrates. Key Engineering Materials. 443, 693-698.

Torr, G.R., 1984. The acoustic radiation force. American Journal of Physics. 52, 402-408.

Vladoiu, I., Stafe, M., Negutu, C., Popescu, I.M., 2008. The dependence of the ablation rate of metals on nanosecond laser fluence and wavelength. Journal of Optoelectronics and Advanced Materials. 10, 3177-3181.

Wu, B., 2014. Ultrasound-assisted water-confined laser micromachining. U.S. Patent Application, Serial No. 14/212,876, filed on March 14, 2014 (the relevant provisional patent application was filed on March 15, 2013, with the application number: 61/787,902).

Zheng, H.Y., Huang, H., 2007. Ultrasonic vibration-assisted femtosecond laser machining of microholes. Journal of Micromechanics and Microengineering. 17, N58-N61. 